\title{
REFLEXIVITY OF OPERATOR SPACES
}

\author{
J. M. BAKER
}

\begin{abstract}
For reflexive Banach spaces $E$ and $F$ (with $E$ or $F$ having the approximation property), the space of opeartors from $E$ into $F$ (the inductive tensor product of $E^{*}$ with $F$ ) is reflexive if and only if the operator space coincides with the inductive tensor product of $E^{*}$ with $F$. Consequently, $E$ must be finite-dimensional if either the projective tensor product of $E$ with $E^{*}$ is reflexive, or the inductive tensor product of $E$ with $E^{*}$ is reflexive and $E$ has the approximation property.
\end{abstract}

Introduction. Throughout, $E$ and $F$ will be reflexive Banach spaces, $E \otimes_{\epsilon} F$ the Banach space tensor product of $E$ and $F$ under the least cross norm $\epsilon$ (operator norm, norm giving the inductive topology), and $E \otimes_{\pi} F$ the Banach space tensor product of $E$ and $F$ under the greatest cross norm $\pi$ (nuclear norm, norm giving the projective topology).

In this note we give solutions to two unsolved problems:

A. Does there exist an infinite-dimensional Banach space $E$ such that $E \otimes_{\epsilon} E^{*}$ or $E \bigotimes_{\pi} E^{*}$ is reflexive?

B. Characterize those spaces $L(E, F)$ of operators from $E$ into $F$ which are reflexive.

Problem A was posed in [3] and is answered negatively for the $\pi$ norm, and for the $\epsilon$ norm if $E$ has the approximation property (a.p.). Grothendieck [2] characterized

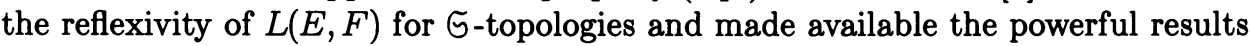
required for the norm topology when both $E$ and $F$ have the a.p. [2]. Thus, for that case, problem B was solved in [3] and [5]. Subsequently, Holub succeeded in removing the a.p. condition from one of the spaces and tied problem $B$ to the theory of operators which attain their norm [4]. The characterization offered below is a.p.-independent.

\section{Results.}

THEOREM 1. For reflexive Banach spaces $E$ and $F, L(E, F)$ is reflexive if and only if $L(E, F)=E^{*} \otimes_{\epsilon} F$.

PROOF. The sufficiency follows from [4, p. 176] since the proof therein of (ii) implies (i) does not assume that either space has the a.p.

If $L(E, F)$ is reflexive, then its closed subspace $E^{*} \otimes_{\epsilon} F$ is reflexive, and since $\epsilon$ is a reflexive cross norm, $\left(E^{*} \otimes_{\epsilon} F\right)^{*}=E \otimes_{\epsilon^{0}} F^{*}[6]$. From $[1$, p. 72, 18] it follows that $E \otimes_{\pi} F^{*} \mid\left(E^{*} \otimes_{\epsilon} F\right)^{0}=E \otimes_{\epsilon^{0}} F^{*}$, where the polar is taken in $E \otimes_{\pi} F^{*}=$ $L(E, F)^{*}$. But

$$
\left[E \otimes_{\pi} F^{*} \mid\left(E^{*} \otimes_{\epsilon} F\right)^{0}\right]^{*}=\left(E^{*} \otimes_{\epsilon} F\right)^{00},
$$

Received by the editors August 25, 1981.

1980 Mathematics Subject Classification. Primary 46B10, 46A32.

(c) 1982 American Mathematical Society 0002-9939/81/0000-1059/\$01.75 
where the second polar is taken in $L(E, F)$. Thus

$$
\left(E^{*} \otimes_{\epsilon} F\right)^{00}=\left(E \otimes_{\epsilon^{0}} F^{*}\right)^{*}=:=E^{*} \otimes_{\epsilon} F .
$$

However,

$$
L(E, F) \mid E^{*} \otimes_{\epsilon} F=\left(E^{*} \otimes_{\epsilon} F\right)^{00}
$$

and the result follows.

CoRollary 2. $E \otimes_{\pi} F^{*}$ is reflexive if and only if $L(E, F)=E^{*} \bigotimes_{\epsilon} F$. Consequently, if $E \otimes_{\pi} E^{*}$ is reflexive, $E$ is finite dimensional.

PROOF. The first statement is a simple consequence of the theorem. If $L(E, E)=$ $E^{*} \otimes_{\epsilon} E$, then the identity operator on $E$ is compact and $E$ must then be finite dimensional.

The following is accessible via several powerful theorems in [2]. The argument is actually shorter, however, by using a well-known result on polars and one of those theorems.

THEOREM 3. If $E$ and $F$ are Banach spaces and one has the a.p., then $E^{*} \otimes_{\epsilon} F$ is reflexive if and only if $L(E, F)=E^{*} \bigotimes_{\epsilon} F$.

Proof. Since either $E$ or $F$ has the a.p., the canonical map $E \otimes_{\pi} F^{*} \rightarrow$ $\left(E^{*} \otimes_{\epsilon} F\right)^{*}$ is an isometry into the second space [2, p. 179], and since $E^{*} \otimes_{\epsilon} F$ is reflexive, $\left(E^{*} \otimes_{\epsilon} F\right)^{*}=E \otimes_{\epsilon^{0}} F^{*}[6]$. Thus,

$$
\left(E \otimes_{\epsilon^{0}} F^{*}\right)^{*} \mid\left(E \otimes_{\pi} F^{*}\right)^{0}=\left(E \otimes_{\pi} F^{*}\right)^{*}=L(E, F),
$$

where the polar is taken in $\left(E \otimes_{\epsilon^{0}} F^{*}\right)^{*}=E^{*} \otimes_{\epsilon} F$. Since the polar is $\{0\}$, $E^{*} \otimes_{\epsilon} F=L(E, F)$.

There immediately follows

COROLlaRY 4. If $E$ or $F$ has the a.p., $E^{*} \otimes_{\epsilon} F$ is reflexive if and only if $E \otimes_{\pi} F^{*}$ is reflexive. Consequently, if $E^{*} \bigotimes_{\epsilon} E$ is reflexive then $E$ is finite dimensional.

Corollaries 2 and 3 thus solve problem III of [3] for the $\pi$ norm, and the $\epsilon$ norm if $E$ has the a.p.

THEOREM 5. Let $E$ and $F$ be reflexive Banach spaces one of which has the a.p. The following are equivalent:

(i) $L(E, F)$ and $L(F, E)$ are reflexive;

(ii) every operator $T: E \rightarrow F$ and $S: F \rightarrow E$ is compact;

(iii) one of $E^{*} \otimes_{\epsilon} F$ or $E \otimes_{\pi} F^{*}$ is reflexive, and one of $E \otimes_{\epsilon} F^{*}$ or $E^{*} \otimes_{\pi} F$ is reflexive;

(iv) all of $E^{*} \bigotimes_{\epsilon} F, E \otimes_{\pi} F^{*}, E \otimes_{\epsilon} F^{*}$, and $E^{*} \bigotimes_{\pi} F$ are reflexive.

Proof. The equivalence of (i) and (ii) was shown in [4]. Theorem 3 and Corollary 4 give the equivalence of (i), (iii), and (iv).

REMARK. We will say that the pair of spaces $E$ and $F$ of Theorem 5 has $\mathrm{P}$ if they have any one of the four equivalent properties. The existence of a pair of infinite-dimensional spaces having property $\mathrm{P}$ is an unsolved problem. This problem was first posed in the setting of statement (ii) by Pelczynski at the Sopot conference for arbitrary $E$ and $F$, and in the setting of (i) by Holub [3]. 


\section{REFERENCES}

1. N. Dunford and J. T. Schwartz, Linear operators. I, Interscience, New York, 1958.

2. A. Grothendieck, Produits tensoriels topologique et espaces nucléaires, Mem. Amer. Math. Soc., no. 16, Amer. Math. Soc., Providence, R. I., 1955.

3. J. R. Holub, Hilbertian operators and reflexive tensor products, Pacific J. Math. 36 (1971), 185194.

4. - Reflexivity of $L(E, F)$, Proc. Amer. Math. Soc. 39 (1973), 175-177.

5. W. Ruckle, Reflexivity of $L(E, F)$, Proc. Amer. Math. Soc. 34 (1972), 171-174.

6. R. Schatten, A theory of cross spaces, Ann of Math. Studies, no. 26, Princeton Univ. Press, Princeton, N. J., 1950.

Department of Mathematics, Western Carolina University, Cullowhee, NORTH CAROLINA 28723 